\title{
VLT/NACO adaptive optics imaging of the TY CrA system
}

\section{A fourth stellar component candidate detected}

\author{
G. Chauvin ${ }^{1}$, A.-M. Lagrange ${ }^{1}$, H. Beust ${ }^{1}$, T. Fusco ${ }^{2}$, D. Mouillet ${ }^{3}$, F. Lacombe $^{4}$, P. Pujet ${ }^{5}$, G. Rousset ${ }^{2}$, \\ E. Gendron ${ }^{4}$, J.-M. Conan ${ }^{2}$, D. Bauduin ${ }^{4}$, D. Rouan ${ }^{4}$, W. Brandner ${ }^{6}$, R. Lenzen ${ }^{6}$, \\ N. Hubin ${ }^{7}$, and M. Hartung ${ }^{6}$
}

1 Laboratoire d'Astrophysique, Observatoire de Grenoble, 414 rue de la piscine, 38400 Saint-Martin d'Hères, France

2 ONERA, BP52, 29 avenue de la Division Leclerc, 92320 Châtillon Cedex, France

${ }^{3}$ Laboratoire d'Astrophysique, Observatoire Midi-Pyrénées, 57 Av. d'Azereix, BP 826, 65008 Tarbes Cedex, France

${ }^{4}$ Laboratoire D'Études Spatiales et d'Instrumentation en Astrophysique, Observatoire de Paris, Bat. 16, 5 Place J. Jansen, 92195 Meudon, France

5 Canada-France-Hawaii Telescope Corporation, PO Box 1597, Kamuela, HI96743, USA

${ }^{6}$ Max-Planck-Institut für Astronomie, Königstuhl 17, 69117 Heidelberg, Germany

7 ESO, Karl-Schwarzschild-Str. 2, 85748 Garching, Germany

Received 17 February 2003 / Accepted 13 April 2003

\begin{abstract}
We report the detection of a possible subsolar mass companion to the triple young system TY CrA using the NACO instrument at the VLT UT4 during its commissioning. Assuming for TY CrA a distance similar to that of the close binary system HD 176386, the photometric spectral type of this fourth stellar component candidate is consistent with an $\sim \mathrm{M} 4$ star. We discuss the dynamical stability of this possible quadruple system as well as the possible location of dusty particles inside or outside the system.
\end{abstract}

Key words. binaries: eclipsing - stars: pre-main sequence - stars: low-mass - circumstellar matter

\section{Introduction}

The CrA dark cloud has been extensively studied from $\mathrm{X}$ ray (Neuhäuser et al. 2000) to IR (Wilking et al. 1997) and radio wavelengths as one of the closest star forming regions. Nearby clouds such as the reflection nebula around R CrA greatly help understanding the stellar formation processes and can be used to compare general tendancies in various star forming regions (see e.g. Chen et al. 1997). This small cloud is now known to host dozens of young stars, mostly low mass stars and brown dwarfs, plus a few high mass stars. Among the latters, TY CrA (B9) is the one of the brightest at optical and near IR, together with RCrA (A5), HD 176386 (A0) and T CrA (F0). As other peculiar massive multiple systems HR 5999 with a $\mathrm{T}$ Tauri companion (Stecklum et al. 1995) or the eclipsing binary EK Cep (Claret 1995b), TY CrA deserves particular attention as it adresses the question of massive star formation.

The distance of the TY CrA system is presently unknown. However, at 57" away, the binary system HD 176386 shares common proper motion with TY CrA (Teixeira et al. 2000). We can then reasonably assume that these two systems are physically bound and then consider for TY CrA the Hipparcos

Send offprint requests to: G. Chauvin,

e-mail: gchauvin@obs.ujf-grenoble.fr distance of $136_{-19}^{+25} \mathrm{pc}$ measured for HD 176386 (as assumed by Siebenmorgen et al. 2000).

TY CrA has been identified and characterized by Corporon et al. (1994) as an eclipsing spectroscopic binary (SB) Pre Main Sequence (PMS) system. Two years later, Casey et al. (1995) and Corporon et al. (1996) showed that TY CrA is a triple SB system, the first one detected among young and massive Herbig stars. Eclipsing binary stars are very precious as they allow a precise determination of the star masses. Coupled to evolutionary tracks, they help deriving ages for the system. Both mass and age are crucial parameters for studies of stellar systems dynamics and stability and provide thus valuable constraints on binary system early evolution processes (see e.g. Prato et al. 2002). In the case of TY CrA, the masses have been found to be $M_{1}=3.0 M_{\odot}, M_{2}=1.6 M_{\odot}$ and $M_{3}=1.2-1.4 M_{\odot}$ (Casey et al. 1998; Corporon et al. 1996). The secondary and tertiary components are very close to the primary, with semi major axes of respectively $14 R_{\odot}(0.065 \mathrm{AU})$ and $1-1.5 \mathrm{AU}$. Using evolutionary tracks of Swenson et al. (1994); Claret (1995a); D'Antona et al. (1994), Casey et al. (1998) found an age of order $3 \mathrm{Myr}$ for the system, confirming the estimation done by Beust et al. (1997). This figure may be compared with the age of 4 Myr recently derived by Palla \& Stahler (2001). 
Table 1. NACO observations of TY CrA and SAO210858: instrumental set-up and observations logs. Camera S27 and S13 respectively provide 27.0 and 13.4 mas/pixel sampling. DIT and NDIT correspond respectively to the exposure integration time and the number of exposures.

\begin{tabular}{llllllll}
\hline \hline Star & $\begin{array}{l}\text { Central wavelength } \\
(\mu \mathrm{m})\end{array}$ & $\begin{array}{l}\text { Bandwidth } \\
(\mu \mathrm{m})\end{array}$ & $\begin{array}{l}\text { DIT } \\
(\mathrm{s})\end{array}$ & NDIT & Camera & WFS & Dichroic \\
\hline TY CrA & 2.166 & 0.023 & 0.6 & 100 & S27 & VIS & VIS \\
TY CrA & 1.257 & 0.014 & 1 & 25 & S27 & VIS & VIS \\
SAO 210858 & 2.166 & 0.023 & 0.5 & 50 & S13 & VIS & VIS \\
SAO 210858 & 1.257 & 0.014 & 1 & 25 & S13 & VIS & VIS \\
\hline
\end{tabular}

The TY CrA system exhibits IR excesses indicative of circumstellar (CS) dust (Cruz-Gonzalez et al. 1984; Wilking et al. 1985; Bibo et al. 1992). At least part of this dust is cold, but the CS material has not been resolved sofar.

The richness of the TY CrA system encouraged us to conduct Adaptive Optics (AO) imaging observations with NACO at the VLT UT4 in the course of the instrument commissioning. A faint visual additional companion candidate, unknown sofar, was discovered close to the unresolved triple system. We present in Sect. 2 the results of the VLT/NACO AO observations. In Sect. 3, we discuss the photometry of the additional companion candidate to TY CrA to test if this object is bound and to determine the corresponding stellar parameters. In Sect. 4, we finally discuss the dynamical status of the possible quadruple system, i.e. the possible existence of a Kozai resonance between the possible fourth stellar component and the three others. We also discuss the possible location of dusty particles in this system.

\section{Observations and results}

Near IR images of the TYCrA system (RA $=19: 01: 41$, $\delta=-36: 52: 34$ (J2000), $V=9.4$, Spectral Type Herbig Be) have been recorded on March 31th 2002 with NACO (Lagrange et al. 2002; Lenzen et al. 2002). NACO is composed of NAOS, the first Adaptive Optics System on the VLT (Rousset et al. 2002) and on the other hand of CONICA, a 1-5 $\mu \mathrm{m}$ imaging, coronographic, spectroscopic and polarimetric instrument (Lenzen et al. 1998).

For these observations, we selected NACO's visible wavefront sensor. With the adequate dichroic, the visible light was directed to NAOS and all the IR light towards CONICA. The CONICA detector is an Aladdin InSb $1024 \times 1024$ pixel array. The cameras used were either S13 or S27 which provide respectively 0.0134 and 0.0270 arcsec/pixel samplings adapted to the observing wavelengths. The star SAO 210858 (RA = 19:03:12, $\delta=-37: 29: 11$ (J2000), A5, $V=10.0)$ located close to TY CrA was also recorded to get PSF calibrations as close as possible in time to TY CrA observations. The detail of the instrumental set-up is given in Table 1. Although the observations were performed under poor seeing conditions (1.3-1.8'), a faint additional object is detected at $0.3^{\prime \prime}$ from the unresolved triple system TY CrA. Figure 1 presents the AO images obtained at $1.257 \mu \mathrm{m}$ and $2.166 \mu \mathrm{m}$. The observations were then deconvolved using MISTRAL algorithm (Conan et al. 2000; Fusco et al. 1999). The results are reported in Table 2.
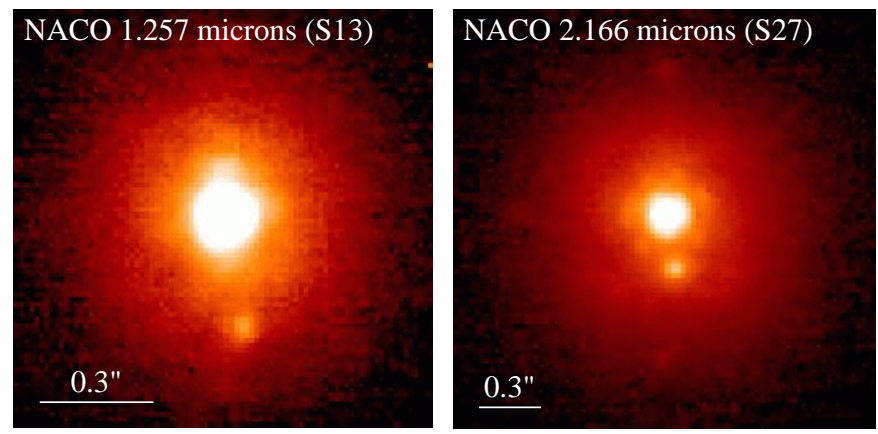

Fig. 1. VLT/NACO AO imaging of the unresolved triple system TY CrA and the fourth stellar component candidate detected at $0.3^{\prime \prime}$ at $1.257 \mu \mathrm{m}$ and $2.166 \mu \mathrm{m}$. North is up and east is left.

Table 2. MISTRAL deconvolution results. Contrast and relative position between the unresolved triple system and the fourth stellar component candidate observed at $1.257 \mu \mathrm{m}$ and $2.166 \mu \mathrm{m}$.

\begin{tabular}{lllll}
\hline \hline Source & $\begin{array}{l}\Delta[1.257] \\
(\mathrm{mag})\end{array}$ & $\begin{array}{l}\Delta[2.166] \\
(\mathrm{mag})\end{array}$ & $\begin{array}{l}\text { Separation } \\
(\operatorname{arcsec})\end{array}$ & $\begin{array}{l}\text { PA } \\
(\text { degrees })\end{array}$ \\
\hline TY CrA & $3.5 \pm 0.3$ & $2.8 \pm 0.1$ & $0.294 \pm 0.007$ & $188.5 \pm 1$ \\
\hline
\end{tabular}

\section{Companion or background object?}

As this new companion candidate cannot be identified with one of the known spectroscopic components of the TY CrA triple system (contradiction with well known orbit parameters), the first question is whether the photometry of this object is consistent with the photometry of a fourth, gravitationally bound stellar component and if so, to derive the corresponding stellar parameters. The photometry of the unresolved TY CrA system obtained by de Winter et al. (2001), $J=7.36 \pm 0.1$ and $K=6.57 \pm 0.1$, and the observed contrasts (see Table 2) are used to determine the photometry of the new object. We have made the approximation that the contrasts obtained with the narrow band filters at 1.257 and $2.166 \mu \mathrm{m}$ were respectively similar to the ones with broad bands filters $J$ and $K$. The photometry of the fourth component candidate was then compared to the evolutionary tracks of (Baraffe et al. 1998) for an age of 3.2 and 4 Myr. Regarding the important Hipparcos parallaxe uncertainties, three hypotheses of distances were considered: $161 \mathrm{pc}, 136 \mathrm{pc}$ and $117 \mathrm{pc}$.

In each case, we used then the color-magnitude diagram for the near-IR color $(J-K)$ to determine the expected stellar parameters for the fourth stellar component candidate to TY CrA 


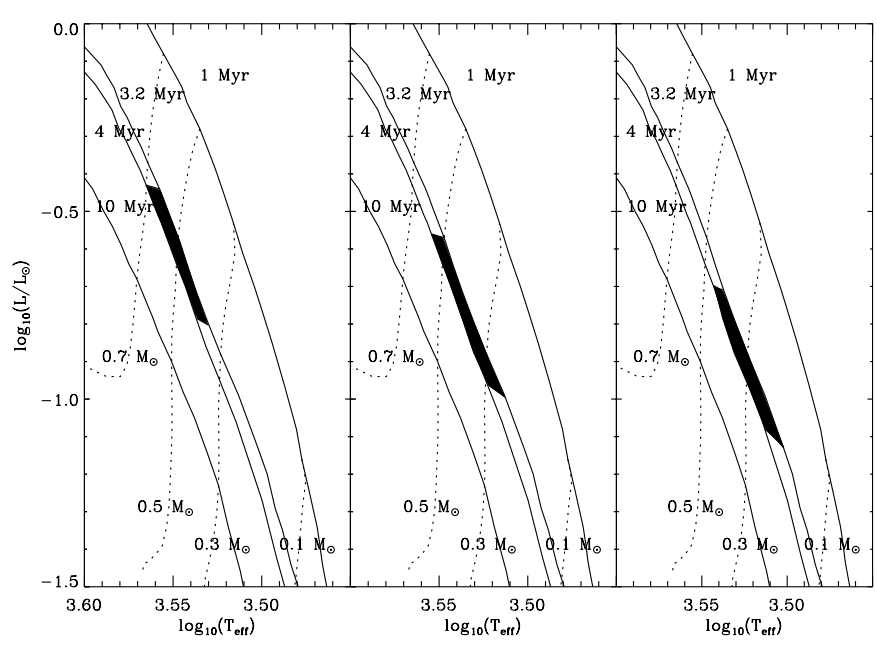

Fig. 2. Evolutionary tracks of Baraffe et al. (1998) with the location of the fourth stellar component candidate to TY CrA based on photometry and assuming an age between 3.2 and $4 \mathrm{Myr}$ for 3 hypothesis of distance: $161 \mathrm{pc}, 136 \mathrm{pc}$ and $117 \mathrm{pc}$ (from left to right).

Table 3. Stellar parameters for the fourth stellar component candidate to TY CrA if bound to the system.

\begin{tabular}{llll}
\hline \hline \multicolumn{4}{c}{ Age of 3.2 Myr } \\
\hline $\begin{array}{l}\text { Distance } \\
\text { to Earth } \\
(\mathrm{pc})\end{array}$ & $\begin{array}{l}\text { Distance } \\
\text { to TY CrA } \\
(\mathrm{AU})\end{array}$ & Mass & $\begin{array}{l}\text { Effective } \\
\text { temperature } \\
(\mathrm{K})\end{array}$ \\
\hline 117 & 35 & $\left(M_{\odot}\right)$ & {$[3178,3445]$} \\
136 & 41 & {$[0.2,0.4]$} & {$[3260,3525]$} \\
161 & 48 & {$[0.25,0.5]$} & {$[3395,3621]$} \\
\hline \hline
\end{tabular}

\begin{tabular}{|c|c|c|c|}
\hline \multicolumn{4}{|c|}{ Age of $4 \mathrm{Myr}$} \\
\hline $\begin{array}{l}\text { Distance } \\
\text { to Earth } \\
\text { (pc) }\end{array}$ & $\begin{array}{l}\text { Distance } \\
\text { to TY CrA } \\
(\mathrm{AU})\end{array}$ & $\begin{array}{l}\text { Mass } \\
\left(M_{\odot}\right)\end{array}$ & $\begin{array}{l}\text { Effective } \\
\text { temperature } \\
(\mathrm{K})\end{array}$ \\
\hline 117 & 35 & {$[0.25,0.45]$} & {$[3270,3492]$} \\
\hline 136 & 41 & {$[0.3,0.57]$} & {$[3340,3584]$} \\
\hline 161 & 48 & {$[0.4,0.7]$} & {$[3452,3674]$} \\
\hline
\end{tabular}

if gravitationally bound. The results are reported in Table 3 and are also overplotted to the evolutionary tracks in Fig. 2. For these three distances, the corresponding spectral types found for an age ranging between 3.2 and 4 Myr are M 1-M4, M 2M 5 and M 3-M 6. Further spectroscopic observations will help to confirm the companionship of this newly detected object and to characterize precisely its spectra.

\section{Dynamical status of the quadruple system?}

We cannot state that the newly discovered object is really bound to the triple system even if the measured colour and brightness are consistent in the frame of the distance and age of the system. Repeated astrometric observations are needed to assert this point. One can however address the effects and consequences if it is physically bound. Hereafter, a $M_{4} \sim 0.4 \pm 0.2 M_{\odot}$ component orbiting the triple system at $\sim 41 \mathrm{AU}$ is considered.
A detailed dynamical modeling of Beust et al. (1997) showed that the stability of the triple system was ensured by tidal effects within the central binary. More specifically, if tidal effects were not present, the secular dynamics of the triple system would be dominated by the Kozai resonance (Kozai 1962; Harrington 1968). In triple stellar system, this phenomenon is characterized by drastic periodic increases of the eccentricity of the central binary, while in the same time the mutual inclination $i$ between the orbit of the central binary and that of the third component would be subject to sharp drops by more than $40^{\circ}$ on very short timescales $\left(<10^{3} \mathrm{yr}\right)$. Active as soon as the inclination $i$ between the two orbits is $\gtrsim 40^{\circ}$ (Söderhjelm 1982 ), only the orbit with the smaller angular momentum (i.e. the inner orbit) is strongly affected (Beust et al. 1997). In the case of TY CrA, the ratio of the third body angular momentum over the central binary angular momentum is $\sim 4.8$. This is enough for initiating a very strong Kozai resonance which would strongly affect the central binary (with a possible physical collision) in the absence of tidal effects.

If now a fourth member is identified, it is worth wondering whether a Kozai resonance exists between this object and the third body around the central binary. This is of valuable interest, because in that case, no tidal effects in the orbit of the third body are to be expected to assure the dynamical stability of the whole system. Actually a specific dynamical study must be initiated and will be the subject of future work. Depending on the mass assumed for the fourth body, and on the semi-major axis and eccentricities values taken (compatible with the measured projected separation), the angular momentum ratio between the orbits of the third and fourth component is expected to range between $\sim 1$ and $\sim 4$. If the mutual inclination is high enough, we may expect a significant Kozai resonance as soon as this ratio is $\gtrsim 2$. We thus cannot definitely conclude at present, but we may suspect that it will be at work and will affect the overall dynamics of the system.

Another important issue is the presence of dust in the system. Cruz-Gonzalez et al. (1984), Wilking et al. (1985) and Bibo et al. (1992) report the detection of a strong farinfrared excess attributed to cold $(95 \pm 10 \mathrm{~K})$ dust located at about $900 \mathrm{AU}$ from the central binary. According to Bibo et al. (1992), the fact that this distance is very large leads to question whether this dust is of CS origin: if there is some CS dust, why is it only at large distance? If the whole system is quadruple, dust particles are very unlikely to be dynamically stable at close distance from the central binary. They might be dynamically stable between the central triple system and the fourth body. This actually depends on the orbit assumed for the fourth component, and on the strength of an eventual Kozai resonance. If present, dust in this area would be warmer ( $\gtrsim 200 \mathrm{~K})$ and generate a mid-infrared excess that has not been detected sofar. In this context, this non-detection is perhaps an indirect indication of dynamical instability in this region and may put some constraints on the dynamical status of the system. Dust is more safely expected to be found orbiting the four-body system, but not closer to 2-3 times the distance of the fourth body, i.e. at least $100 \mathrm{AU}$, and probably more if radiation pressure from the central binary is taken into account. The structure of this dust component (circum-quadruple disk or spherical shell?) is an 
open question and should depend actually on the dynamical behavior of the four-body system. Obviously a dedicated dynamical study is required.

\section{Conclusion}

We have presented results of VLT/NACO adaptive optics imaging of the TY CrA system. A fourth stellar component candidate is detected at $\sim 0.3^{\prime \prime}$ from the unresolved triple system. It is not possible at present time to conclude whether this fourth component is physically bound to the system. If this is the case, this object is likely to be a $\sim \mathrm{M} 4$ dwarf based on the evolutionary tracks of Baraffe et al. (1998) for an age ranging between 3.2 and $4 \mathrm{Myr}$ and a distance of $136_{-19}^{+25} \mathrm{pc}$. If so, we can then expect the possible existence of a Kozai resonance between the orbit of the fourth component and the third body around the central binary, which could affect the stability of the whole system. Dust particles might be also dynamically unstable between the third and fourth component. The only detection of a strong infrared excess attributed to cold dust orbiting the four-body system may be an indirect indication of dynamical instability in this region. Further dynamical study must be initiated to investigate the status of this possible quadruple system and of the possible location of dust inside or outside the system.

Acknowledgements. We would like to thank the staff of the ESO paranal observatory and the NAOS/CONICA team for their work on the instrument (without them, this work could not have been carried on) and particulary Françoise Crifo for her precious comments.

\section{References}

Baraffe, I., Chabrier, G., Allard, F., \& Hauschildt, P. H. 1998, A\&A, 337, 403

Beust, H., Corporon, P., Siess, L., Forestini, M., \& Lagrange, A.-M. 1997, A\&A, 320, 478

Bibo, E. A., Thé, P. S., \& Dawanas, D. N. 1992, A\&A, 277, 439
Casey, B. W., Mathieu, R. D., Suntzeff, N. B., \& Walter, F. M. 1995, AJ, 109, 2156

Casey, B. W., Mathieu, R. D., Luiz Paulo, R. V., Andersen, J., \& Suntzeff, N. B. 1998, AJ, 115, 1617

Chen, H., Grenfell, T. G., Myers, P. C., \& Hughes, J. D. 1997, ApJ, 478, 295

Claret, A. 1995, A\&A, 109, 441

Claret, A., Giménez, A., \& Martín, E. L. 1995, A\&A, 302, 741

Conan, J.-M., Fusco, T., Mugnier, L., et al. 2000, Proc. SPIE, 4007, 913, ed. P. L. Wizinowich

Corporon, P., Lagrange, A.-M., \& Bouvier, J. 1994, A\&A, 282, L21

Corporon, P., Lagrange, A.-M., \& Beust, H. 1996, A\&A, 310, 228

Cruz-Gonzalez, I., McBreen, B., \& Fazio, G. G. 1984, ApJ, 279, 679

D’Antona, F., \& Mazzitelli, I. 1994, ApJS, 90, 467

de Winter, D., Van den Ancker, M. E., Maira, A., et al. 2001, A\&A, 380,609

Fusco, T., Véran, J.-P., Conan, J.-M., \& Mugnier, L. 1999, A\&AS, 134, 193

Harrington, R. S. 1968, AJ, 73, 190

Kozai, Y. 1962, AJ, 67, 591

Lagrange, A.-M., Mouillet, D., Beuzit, J.-L., et al. 2002, SPIE, in press Lenzen, R., Hofmann, R., Bizenberger, P., \& Tusche, A. 1998, SPIE, 3354

Lenzen, R., Hartung, M., Brandner, et al. 2002, SPIE, 4841

Neuhäuser, R., Walter, F. M., Covino, E., et al. 2000, A\&AS, 146, 323

Palla, F., \& Stahler, S. W. 2001, ApJ, 553, 299

Prato, L., Simon, M., Mazeh, T., et al. 2002, ApJ, 569, 863

Rousset, G., Lacombe, F., Puget, P., et al. 2002, SPIE, 4007

Siebenmorgen, R., Prusti, T., Natte, A., \& Müller, T. G. 2000, A\&A, 361,258

Söderhjelm, S. 1982, A\&A, 107, 54

Stecklum, B., Eckart, A., Henning, Th., \& Löwe, M. 1995, A\&A, 296, 463

Swenson, F. J., Faulkner, J., Roger, F. J., \& Iglesias, C. A. 1994, ApJ, 425, 286

Teixeira, R., Ducourant, C., Sartori, M. J., et al. 2000, A\&A, 361, 1143

Wilking, B. A., Harvey, P. M., Joy, M., Hyland, A. R., \& Jones, T. J. 1985, ApJ, 293, 165

Wilking, B. A., McCaughrean, M. J., Burton, M. G., et al. 1997, AJ, 114,2029 